\title{
Role of Multi-Detector CT in Diagnosis of Splenic Lesions
}

\author{
Medhat M. Refaat, Osama T. Galal, Sara A. Abdullatif
}

\begin{abstract}
Department of diagnostic and interventional radiology, Benha faculty of medicine, Banha University, Egypt.

Correspondence to: Sara A. Abdullatif, Department of diagnostic and interventional radiology, Benha faculty of medicine, Banha University, Egypt.
\end{abstract}

Email:

sara.almoneeb@gmail.com

Received: 23 June 2021

Accepted: 26 November 2021

\begin{abstract}
Background: A wide range of splenic lesions may be encountered in clinical practice. The aim of the study is to highlight the role of MDCT in the differential diagnosis of splenic lesions. Methods: This retrospective study was carried out on 20 patients presented to radiology department at Benha University Hospitals for diagnosis, during the period from January 2019 to January 2020. Results: Among the 20 patients included in this study; 9 of them were males and the other 11 were females with no significant relation between the sex and the pathology, 5 of the patients were diagnosed as cystic lesions while 11 cases were diagnosed as solid lesions as follow; 4 patients were diagnosed as hemangioma, 6 patients were diagnosed as lymphoma, And one patient was diagnosed as splenic metastases, And 4 patients were diagnosed as others as follow; 3 patients were diagnosed as infarction, one patient was diagnosed as splenic lacerations.
\end{abstract}

Conclusion: CT is a good screening modality for the spleen and its findings are useful to differentiate benign from malignant splenic lesions, which are characterized by mainly solid nature of the splenic mass and presence of underlying malignancy.

Keywords: multi-detector; CT; splenic lesions

\section{Introduction}

A wide range of splenic lesions may be encountered in clinical practice. Although most of the incidentally detected splenic lesions are benign, differential diagnosis of benign and malignant lesions is still a radiological challenge due to overlapping imaging features. However, since most of the patients with splenic lesions who undergo abdominal CT scans manifest various types of underlying disease 
including malignancy, it is essential to provide reasonable differential diagnosis for appropriate management. (1)

The spleen is the largest organ in the reticulo-endothelial system. It is located posterolaterally in the left upper part of the abdominal cavity. It is a "coffee bean shaped" organ with the posterior border is rounded, but the anterior border is notched. The diaphragmatic surface is convexly curved to fit the concavity of the diaphragm while the visceral surface is related to the stomach, left kidney, left suprarenal gland and left colic flexure. (2)

All the splenic vessels, lymphatics and nerves enter and leave the organ in the hilum. Centrally situated in the medial surface. The hilum is the only part of spleen not covered by peritoneum. Peritoneal folds or ligaments attach the spleen to adjacent viscera and the abdominal wall. (3)

Most splenic lesions are found incidentally. Often these lesions are asymptomatic when small and may become painful as they enlarge due to mass effect and stretching of the splenic capsule. Surgical consultation should be considered when abdominal pain is potentially attributable to a splenic mass. (4)
The purpose of the study is to highlight the role of MDCT in the differential diagnosis of splenic lesions.

\section{Patients and methods}

This retrospective study was carried out on 20 patients presented to Radiology Department at Benha University Hospitals for diagnosis, during the period from January 2019 to January 2020.

The patients were included according to the following criteria;

\section{Inclusion criteria:}

1. Patients who are referred after ultrasound examination without definite diagnosis.

2. Patients with symptoms of left upper abdominal pain

3. Anemia

4. Fatigue.

5. Frequent infections.

6. Easy bleeding.

7. Abnormal blood count (CBC)

\section{Exclusion criteria:}

1. Pregnancy.

2. Obese patients.

3. Poor patient cooperation. 


\section{Data procession:}

Through-full clinical history taking and previously available examination.

\section{Ethical committee approval:}

- Approval of the ethics committee of researchers of Benha university.

- An informed consent was taken from all the participants before taking any data or doing any imaging techniques.

- The consent contains:

1. Simple explanation of the aim of the study to the patients.

2. No harmful maneuvers were performed or used.

3. All data is considered confidential.

4. All samples will be used in research only.

5. All participants were informed by the result of the study.

6. Signatures or fingerprints of the participants was taken.

\section{Techniques:}

- Computed tomography gives eminent information of the spleen and adjacent structures. After intravenous contrast infusion many pathologic conditions appear even more clearly.
- Patients was asked to fast for six hours prior to the procedure, but they should be well hydrated.

- If the patient has a splenic trauma, CT imaging done with no preparations.

- All patients will be subjected to proper history taking:

o Full clinical assessment including recording of age, sex and clinical presentation.

- Revision of patient's laboratory investigation including $\mathrm{CBC}$, renal function test and liver function test.

$\circ$ Revision of the previous radiological investigation as US and CT.

- All patients underwent Multi-slice CT

- Intravenous contrast was used.

- Patient lies in supine position, if patient has any vertebral column defect as (kyphosis or lordosis) he lies in prone position.

- First, splenic volume assessment was done by measuring splenic index.

- The 'splenic index', which is the product of the width, depth and length of the spleen, has been proposed as an 
indicator for evaluating splenic volume, the normal range is $160-440 \mathrm{~cm}^{3}$.

- We can calculate the volume of spleen manually by using the standard clinical prolate ellipsoid equation for spleen [0.524 $\times$ splenic index]. The average volume of normal spleens is $236.89 \pm$ $77.58 \mathrm{ml}$.

- Thresholds for the identification and grading of splenomegaly from automatic volumetric spleen assessment were introduced. The volumetric thresholds match well with clinical interpretations for splenomegaly and may improve splenomegaly detection compared with splenic cephalocaudal height measurements. Also, it can be used for measuring splenic hilar notch index.

- Second, vascular imaging assessment was done for any abnormality.

- The vascular flow through the cords of the splenic red pulp is variable. This is assumed to be the main reason for the well-documented phenomenon of inhomogeneous enhancement of the normal splenic parenchyma during the first minute of dynamic contrastenhanced imaging. Thus, after approximately $1 \mathrm{~min}$, the splenic tissue achieves a homogeneous appearance again.

- Finally, assessment of splenic parenchyma, On plain CT scans the normal splenic tissue is homogeneous with attenuation values ranging between 40 and 50 Hounsfield units (HU), which is approximately 5-10 HU less than the normal liver.

- All the CT images included portal venous phase, which was used to evaluate splenic lesions. The portal venous scans were acquired at fixed delays (70-80 seconds) after the administration of $1.5-2.0 \mathrm{~mL}$ per kilogram of body weight of nonionic contrast material at a rate of 2.0-3.5 $\mathrm{mL} / \mathrm{sec}$ with a pump via an 18-gauge catheter placed in a peripheral vein.

- We compared the initial CT findings in each case with histopathological findings or other radiological modalities.

\section{Statistical analysis;}

Data were collected, coded, revised and entered to the Statistical Package for Social Science (IBM SPSS) version 20. The data were presented as number and percentages for the qualitative data, mean, standard deviations and ranges for the quantitative 
data with parametric distribution and median with inter quartile range (IQR) for the quantitative data with non-parametric distribution.

\section{Results}

Eleven patients (55\%) were females, 9 patients $(45 \%)$ were males. Mean of age 48.95 with range from 26 to 68 years, table 1.

Ten patients $(50 \%)$ had multiple lesions, of 10 patients $(50 \%)$ had single lesions (figure 1).

Density of all patients were Hypo, 11 patients (55\%) had Enhancement (Table 2).

Thirteen patients (65\%) had Enlarged splenic size, of 7 patients $(35 \%)$ had normal splenic size (Figure 2).

Regarding character of the lesion; 5 patients (25\%) had Cystic, and 11 patients (55\%) had solid lesions (Figure 3).

Regarding associations; 2 patient (10\%) was Cirrhotic liver, 1 patient only (5\%) was septations, of 6 patients $(30 \%)$ was LNs, of 3 patients (15\%) was similar lesions elsewhere, of 1 patient only (5\%) was other traumatic sequale (Figure 4).

Regarding diagnosis; 4 patients (20\%) had Hemangioma, 3 patients (15\%) had Infarction, 7 patients (35\%) had Lymphoma,
1 patient (5\%) had Lacerations, 4 patients (20\%) had Simple cyst and 1 patient (5\%) had Hydatid cyst (Figure 5).

3 patients (15\%) had infarction, 4 patients (20\%) had simple cyst, 4 patients $(20 \%)$ had hemangioma, 6 patients (30\%) had lymphoma, 1 patient (5\%) had lacerations, 1 patient (5\%) had metastatic chest wall fibrosarcoma, 1 patient $(5 \%)$ had hydatid cyst (Table 3).

\section{Case 1: Figure 6}

Male patient aged 3 years presented by blunt trauma to the abdomen. US revealed abdominal collection. CT with IV contrast was done with no preparation. CT images revealed:

- Average size spleen showing two small upper and lower hypodense linear splenic lacerations, measuring about $1.4 \mathrm{~cm}$ and $1.1 \mathrm{~cm}$ in diameters respectively.

- A large retro-peritoneal fluid collection is seen extending from the left subphrenic region to the left anterior pararenal space and creeping anteriorly to the anterior para-pancreatic space, measuring about $11 \times 4 \times 10 \mathrm{~cm}$ along the axial and $\mathrm{CC}$ dimensions, exerting mass effect in the form of displacing the related peritoneum and bowel loops 
anteriorly and the stomach to the right side.

- Also a small pancreatic tail hypodense rather linear density is seen, measuring $1.3 \mathrm{~cm}$ in diameter.

Axial CT cuts: The patient underwent exploration and splenectomy was done.

\section{Case 2: Figure 7}

Female patient aged 48 years presented by abdominal pain with weight loss. Previous history of chest wall fibrosarcoma underwent excision, CT with IV contrast was done after fasting for 6 hours, CT images revealed:

- Markedly enlarged spleen seen studded by innumerable variable sized focal lesions with showing faint homogenous enhancement (arrowed).

- Innumerable enlarged amalgamated para-aortic, celiac, porta-hepatis, peripancreatic and internal and external iliac lymph nodes are noted.
Axial CT cuts: CT interpretation suggest lymphoma. Biopsy from discovered lymph nodes revealed metastasizing primary chest wall malignancy (dermato-fibrosarcoma).

\section{Case 3: figure 8}

Female patient aged 28 years presented by left hypochondrial pain. US revealed multi-locular cystic lesion. CT with IV contrast was done with after 4 hours fasting. CT images revealed:

- Well defined hypodense multiloculated cystic lesion measuring 6.5 $x \quad 5.3 \times \quad 4.5 \mathrm{~cm}$ showing intrinsic calcified septations with no CT evidence of internal post contrast enhancement (arrowed).

Axial and coronal CT cuts; CT interpretation suggest splenic hydatid cyst. No histopathological data and the patient only follow up.

Table (1): Demographic data

\begin{tabular}{llll}
\hline & & No & $\%$ \\
\hline \multirow{2}{*}{ Sex } & Female & 11 & $55.0 \%$ \\
& Male & 9 & $45.0 \%$ \\
\multirow{2}{*}{ Age } & Mean \pm SD & $48.95 \pm 14.68$ & \\
& Range & $26-68$ & \\
\hline
\end{tabular}


Table (2): Density and Enhancement

\begin{tabular}{llll}
\hline & & No & $\%$ \\
\hline Density & Hypo & 20 & $100.0 \%$ \\
Enhancement & No & 9 & $45.0 \%$ \\
& Yes & 11 & $55.0 \%$ \\
\hline
\end{tabular}

Table (3): Histology or Triphasic

\begin{tabular}{llll}
\hline & & No & $\%$ \\
\hline & Infarction & 3 & $15.0 \%$ \\
& Lymphoma & 6 & $30.0 \%$ \\
& Simple cyst & 4 & $20.0 \%$ \\
& Hemangioma & 4 & $20.0 \%$ \\
& Hydatid cyst & 1 & $5.0 \%$ \\
& Lacerations & 1 & $5.0 \%$ \\
& Metastatic chest wall fibrosarcoma & 1 & $5.0 \%$ \\
\hline
\end{tabular}

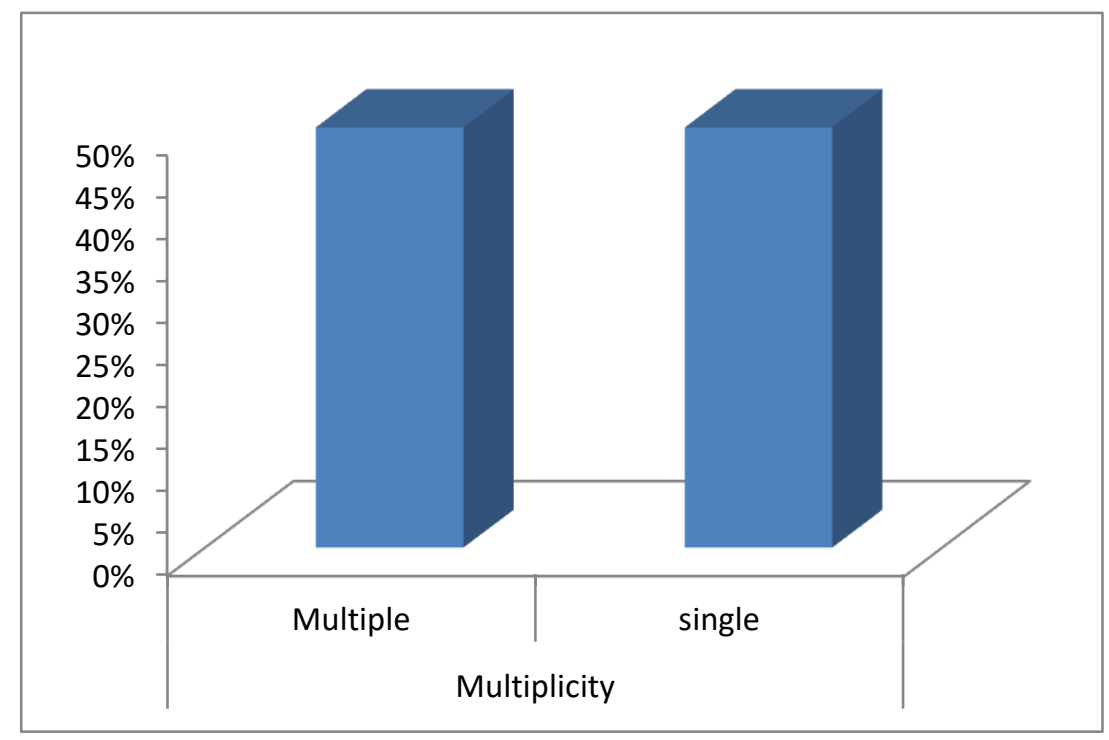

Figure (1): Multiplicity 
Benha medical journal, vol.39, special issue (radiology), 2022

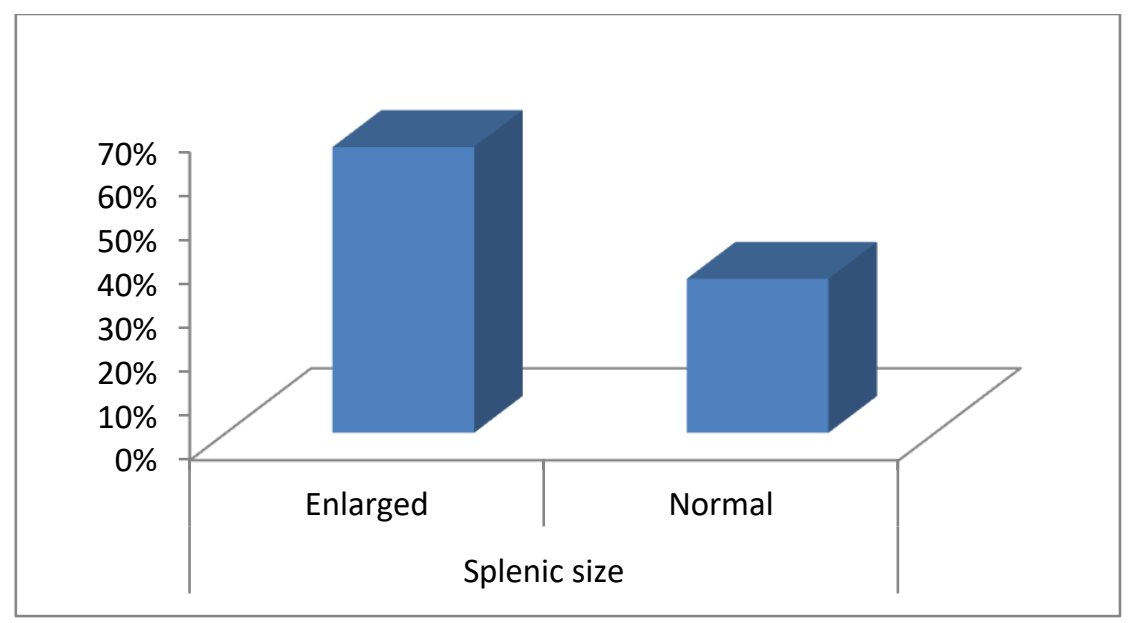

Figure (2): Splenic size

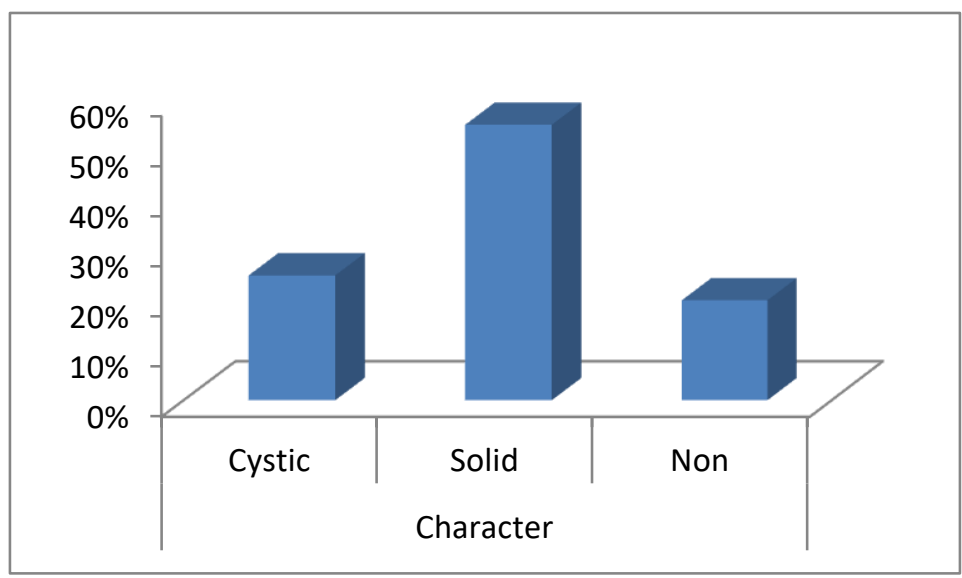

Figure (3): Character

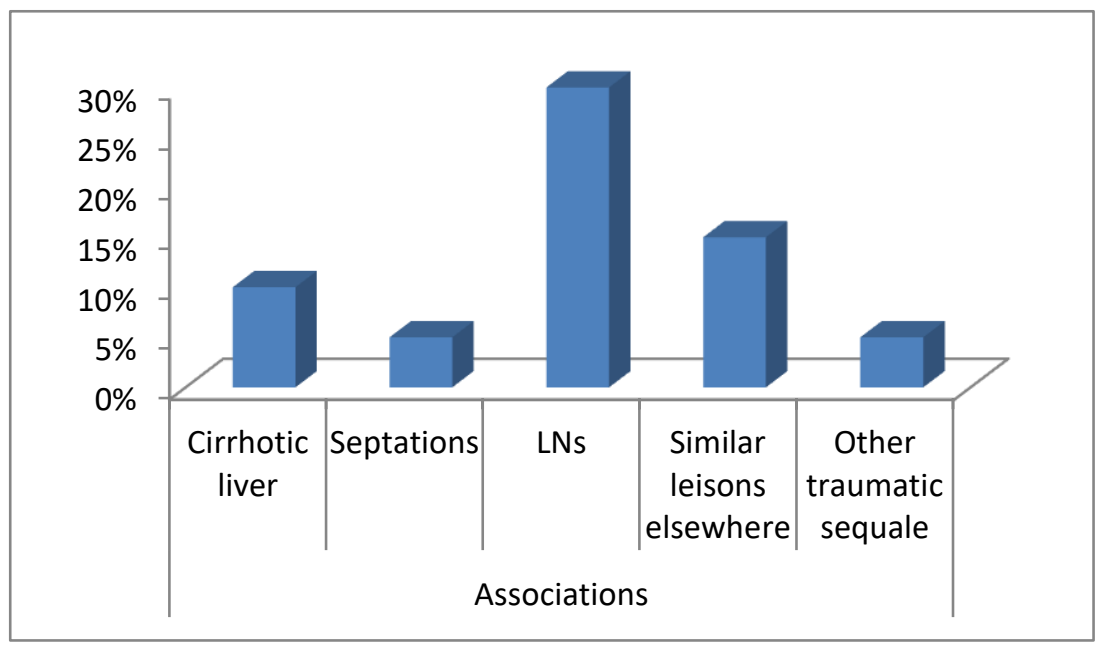

Figure (4): Associations 


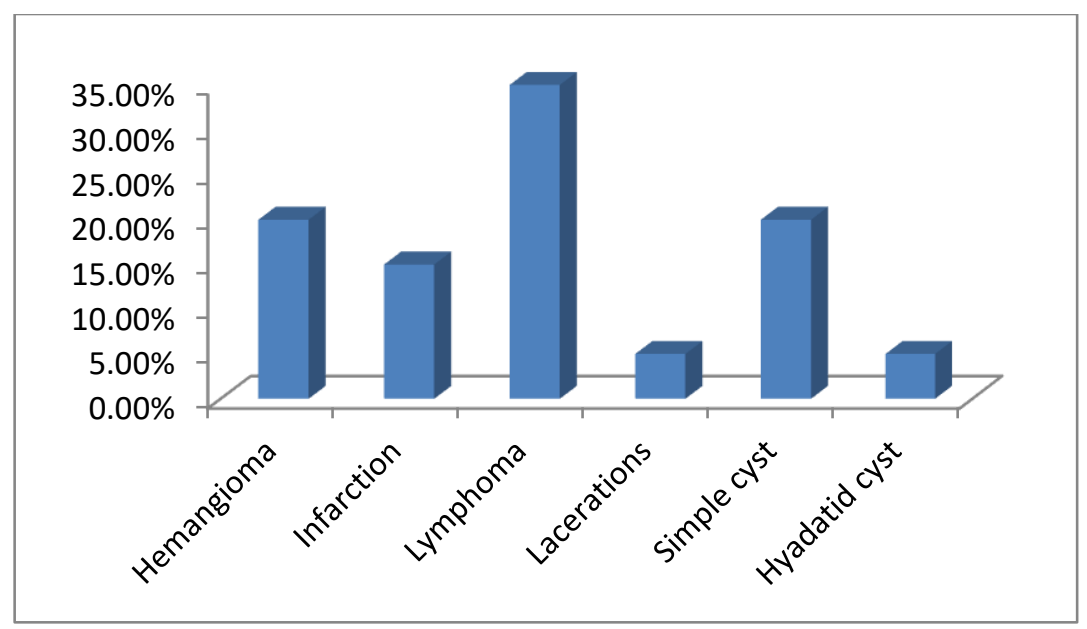

Figure (5): Diagnosis

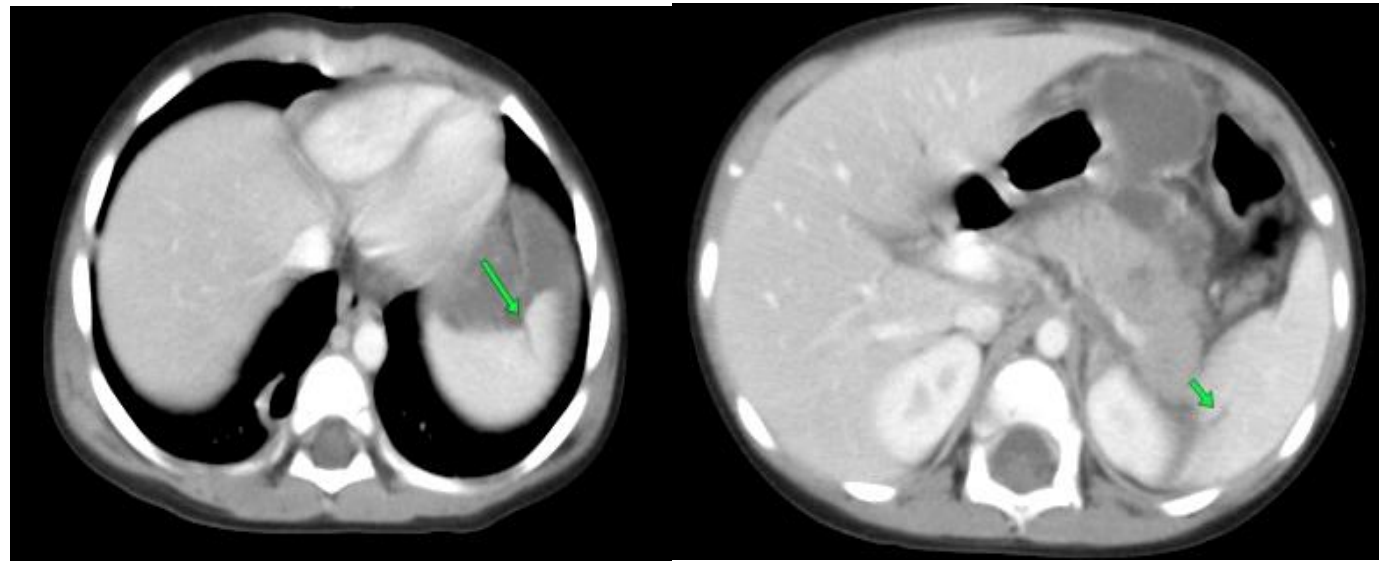

Figure 7

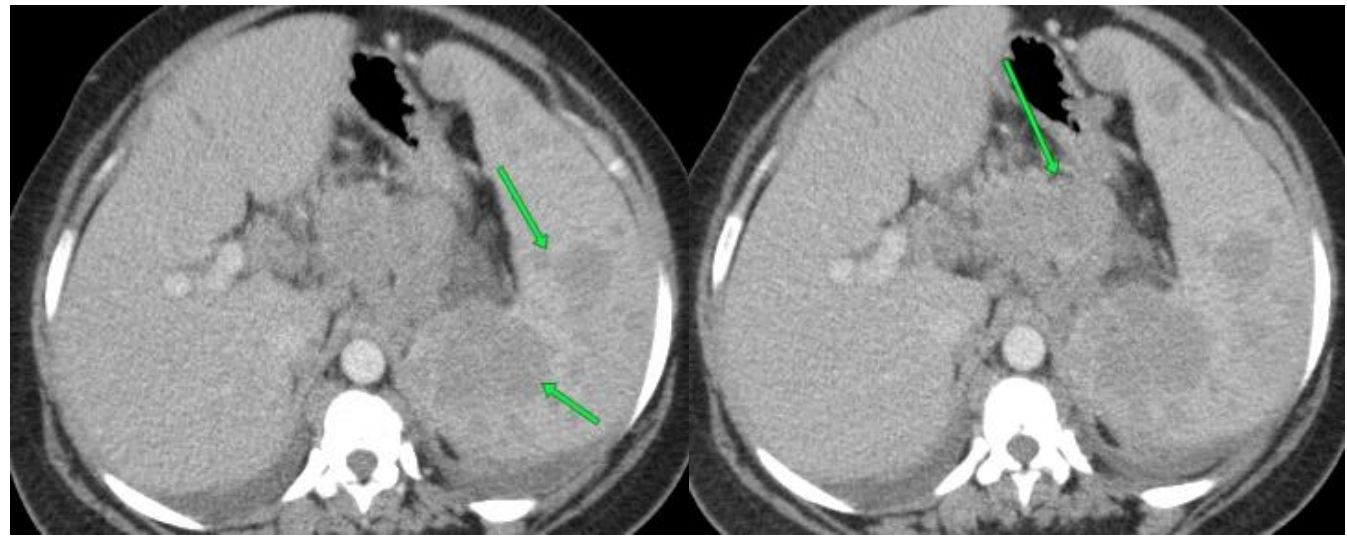

Figure 8 

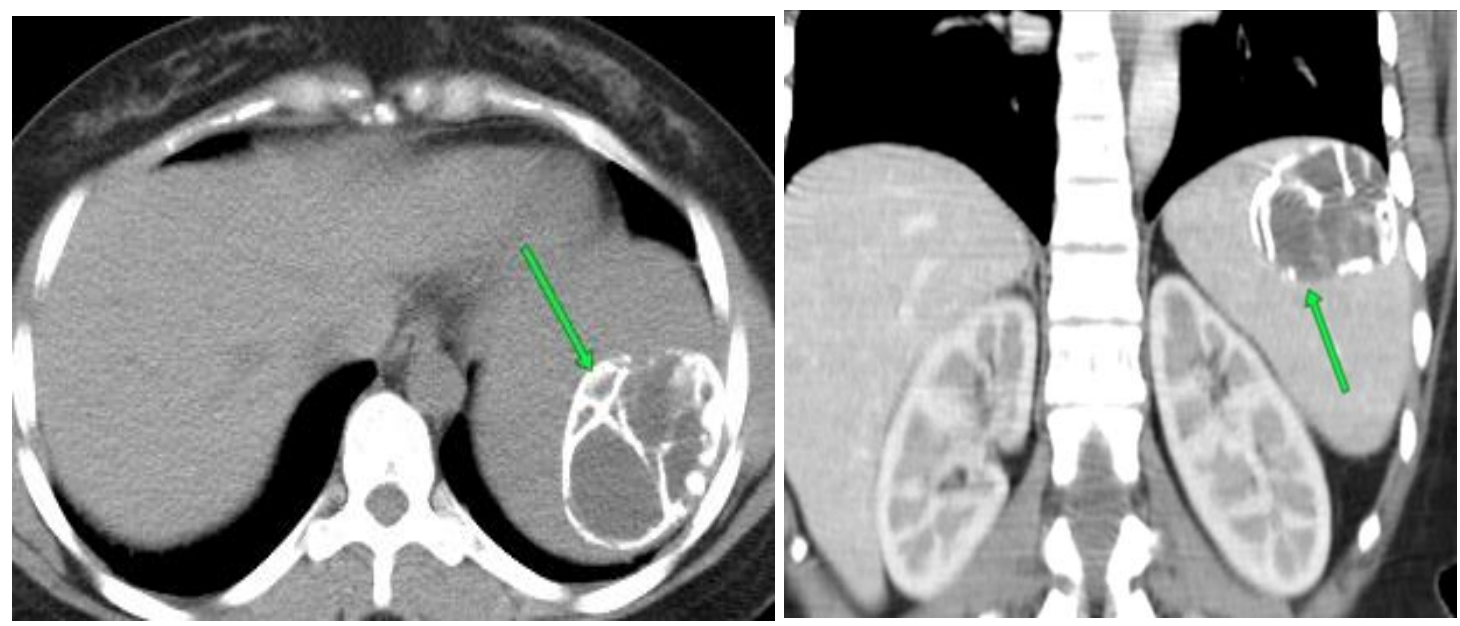

Figure 9

\section{Discussion}

The purpose of our study was to assess the usefulness of using well established simplified algorithm-based approach to the splenic lesions detected on routine computed tomography (CT) of the abdomen $\&$ pelvis.

Among the 20 patients included in this study; 9 of them were males and the other 11 were females with no significant relation between the sex and the pathology. Five of the patients were diagnosed as cystic lesions while 11 cases were diagnosed as solid lesions as follow; 4 patients were diagnosed as hemangioma, 6 patients were diagnosed as lymphoma, one patient was diagnosed as splenic metastases, and 4 patients were diagnosed as others as follow; 3 patients were diagnosed as infarction, one patient was diagnosed as splenic lacerations. In our study all of the cases are seen of hypodense

attenuation. Fifty $\%$ of the patients show single splenic lesion and 50\% show multiple ones.

Ultrasound and Computed tomography are the most important modalities in splenic imaging (5).

Ultrasonography is the first method for splenic imaging, with CT used when necessary for further characterization of splenic lesions (6).

CT attenuation of splenic tissue is homogeneous, typically measuring 40-60HU on non-contrast material enhanced scans (7).

The 'splenic index', which is the product of the width, depth and length of the spleen, has been proposed as an indicator for evaluating splenic volume, the normal range is $160-440 \mathrm{~cm}^{3}$. 
- We can calculate the volume of spleen manually by using the standard clinical prolate ellipsoid equation for spleen $[0.524 \times$ splenic index].The average volume of normal spleens is $236.89 \pm 77.58 \mathrm{ml}$.

- Thresholds for the identification and grading of splenomegaly from automatic volumetric spleen assessment were introduced. The volumetric thresholds match well with clinical interpretations for splenomegaly and may improve splenomegaly detection compared with splenic cephalocaudal height measurements. Also, it can be used for measuring splenic hilar notch index (8).

Splenic lesions tend to be small or infiltrating and vary in size and configuration, making detection difficult, particularly without use of an organ-specific contrast agent. Six major categories are presented: (a) inflammatory disease, (b) splenic cysts, (c) infarction, (d) nonneoplastic and non-infectious diffuse splenic disease, (e) benign tumors, and (f) malignant tumors. (8) Lymphoma is the most common splenic malignancy, and the most common finding of splenic involvement in lymphoma is homogeneous enlargement (9).
The spleen is a relatively rare site for metastatic disease; patients with metastatic lesions in the spleen usually have disease in other sites as well. Breast, lung, ovary, melanoma, and colon cancer are common primary tumors that metastasize to the spleen. Vascular neoplasms of the spleen represent the majority of the nonhematologic/non-lymphoid neoplasms and commonly produce multifocal lesions (10).

Splenic infarction: The causes of splenic infarct are numerous, e. g., embolic, hematologic, splenic vascular disease, pancreatic disease, collagen vascular disease, anatomic abnormalities, and nonhematologic malignancy (11).

Cystic lesions include: post-traumatic, inflammatory and infectious pseudocysts, congenital cysts, lymphangiomas and cystic metastasis. Solid lesions include: both nonneoplastic lesions such as splenic hamartoma, benign tumors such as hemangioma and malignant masses including lymphoma and angiosarcoma (12). The spleen is affected by a variety of conditions but is seldom the site of primary disease. Splenic enlargement is the most common manifestation of disorder of this organ and almost any disease which involves the spleen can cause splenomegaly. 
Splenic size can be readily measured in vivo; however, there is no precise agreement in the literature as to what constitutes the upper limit of normal splenic size. Focal splenic lesions are uncommon; among the most frequent are cysts, benign hemangiomas, and malignant lymphoma. Splenic metastases are uncommon and are usually correlated with widespread malignant disease (13).

\section{Conclusion}

CT is a good screening modality for the spleen and its findings are useful to differentiate benign from malignant splenic lesions, which are characterized by mainly solid nature of the splenic mass and presence of underlying malignancy.

\section{References}

1. Jang S, Kim JH, Hur BY, Ahn SJ, Joo I, Kim MJ, et al. Role of CT in differentiating malignant focal splenic lesions. Korean J Radiol. 2018;19(5):930.

2. Lobe TE. Splenic Disorder. In: Pediatric Surgery. Springer; 2009. p. 593-602.

3. Chaudhry SR, Hulaibi FA, Nahian A, Chaudhry K. Anatomy, Abdomen and Pelvis, Pelvis. StatPearls [Internet]. 2020;
4. Golmohammadzadeh H, Maddah G, Hojjati YS, Abdollahi A, Shabahang H. Splenic cysts: analysis of 16 cases. Casp J Intern Med. 2016;7(3):217.

5. Coffey W, Balasubramanya R. Spleen Imaging. 2020;

6. Vancauwenberghe T, Snoeckx A, Vanbeckevoort D, Dymarkowski S, Vanhoenacker FM. Imaging of the spleen: what the clinician needs to know. Singapore Med J. 2015;56(3):133.

7. Rabushka LS, Kawashima A, Fishman EK. Imaging of the spleen: CT with supplemental MR examination. Radiographics. 1994;14(2):307-32.

8. Prassopoulos P, Daskalogiannaki M, Raissaki M, Hatjidakis A, Gourtsoyiannis N. Determination of normal splenic volume on computed tomography in relation to age, gender and body habitus. Eur Radiol. 1997;7(2):246-8.

9. Saboo SS, Krajewski KM, O'regan KN, Giardino A, Brown JR, Ramaiya N, et al. Spleen in haematological malignancies: spectrum of imaging findings. Br J Radiol. 2012;85(1009):8192.

10. Kamaya A, Weinstein S, Desser TS. Multiple lesions of the spleen: differential diagnosis of cystic and solid lesions. In: Seminars in Ultrasound, CT and MRI. Elsevier; 2006. p. 389-403.

11. Chapman J, Helm TA, Kahwaji CI. Splenic infarcts. 2017;

12. Kaza RK, Azar S, Al-Hawary MM, Francis IR. Primary and secondary neoplasms of the spleen. Cancer Imaging. 2010;10(1):173.

13. Li L, Duan M, Chen W, Jiang A, Li X, Yang $\mathrm{J}$, et al. The spleen in liver cirrhosis: revisiting an old enemy with novel targets. J Transl Med. 2017;15(1):1-10.

To cite this article: Medhat M. Refaat, Osama T. Galal, Sara A. Abdullatif. Role of MultiDetector CT in Diagnosis of Splenic Lesions. BMFJ 2022; 39 (Radiology):107-118. DOI: 10.21608/bmfj.2021.82063.1432. 